\title{
Elevated circulating level of P2X7 receptor is related to severity of coronary artery stenosis and prognosis of acute myocardial infarction
}

\author{
Xiang-Xiang Shi, Kang-Chun Zheng, Pei-Ren Shan, \\ Lei Zhang, Sheng-Jie Wu, Zhou-Qing Huang
}

Cardiac Center, Department of Cardiology, The Key Lab of Cardiovascular Disease of Wenzhou, The First Affiliated Hospital of Wenzhou Medical University, Wenzhou, Zhejiang, People's Republic of China

\begin{abstract}
Background: Acute myocardial infarction (AMI) is a severely life-threatening cardiovascular disease. Previous research has identified an association between the $P 2 X 7$ receptor (P2X7R) and the development of atherosclerosis. However, the correlation of its expression with the clinical prognosis of patients with AMI remains unclear. The present study aimed to investigate the potential role of P2X7R in Chinese patients with AMI.

Methods: Seventy-nine patients with AMI and 48 controls were consecutively enrolled in this prospective observational study. Circulating P2X7R mRNA expression levels and other clinical variables were determined upon admission to the hospital. Patients were followed up for 360 days, and the end-point was considered as the occurrence of major adverse cardiovascular events (MACE).

Results: Circulating P2X7R mRNA expression level in peripheral blood mononuclear cells of patients with AMI were significantly higher than those in controls and had promising diagnostic ability of AMI with an area under the curve of 0.928. Furthermore, P2X7R was demonstrated to be correlated positively with the severity of coronary artery stenosis. Additionally, this is the first study to indicate that higher P2X7R mRNA expression is associated with a higher rate of MACE within 360 days after AMI. Conclusions: The present study showed that the circulating level of P2X7R was elevated in AMI patients and was closely associated with the severity of coronary artery stenosis and prognosis of AMI. (Cardiol J 2021; 28, 3: 453-459)
\end{abstract}

Key words: P2X7R, acute myocardial infarction, coronary artery stenosis, prognosis

\section{Introduction}

Myocardial infarction, a cardiovascular disease that severely threatens human health, is primarily caused by atherosclerosis of the coronary artery. Pathogenesis of atherosclerosis involves the accumulation of lipids and leukocytes in the intima of blood vessel walls leading to plaque, inflammation, and alteration of innate and adaptive immunities $[1,2]$. Rupture of the vulnerable plaque, characterized by a thin fibrous cap and lipid-rich core is the main cause of coronary thrombosis at the site of plaque, leading to acute myocardial infarction (AMI) [3].
Purinergic receptor P2X7 (P2X7R) is a ligandgated cation channel that is significantly expressed in original immune cells. P2X7R is the most compelling member of the purinergic receptor family owing to its unique phenotype and is involved in the production and activation of inflammatory cytokine interleukin-1 beta for the modulation of inflammatory responses [4]. Research has demonstrated that P2X7R is highly expressed in endothelial cells and macrophages infiltrating the atherosclerotic plaques in human carotid arteries [5]. Peng et al. [6] reported that P2X7R plays a crucial role in the development of atherosclerosis through the regu-

Address for correspondence: Zhou-Qing Huang, MD, PhD, Department of Cardiology, the First Affiliated Hospital of Wenzhou Medical University, Zhejiang, 325000, China, e-mail: susiehzq@126.com

This article is available in open access under Creative Common Attribution-Non-Commercial-No Derivatives 4.0 International (CC BY-NC-ND 4.0) license, allowing to download articles and share them with others as long as they credit the authors and the publisher, but without permission to change them in any way or use them commercially. 
Table 1. Characteristics of patients with acute myocardial infarction (AMI) and controls.

\begin{tabular}{lccc}
\hline & AMI $(\mathbf{n}=79)$ & Controls $(\mathbf{n}=48)$ & $P$ \\
\hline Age [years] & $62.9 \pm 12.9$ & $64.8 \pm 10.3$ & 0.393 \\
Male & $65(79.5 \%)$ & $26(54.2 \%)$ & $0.001^{*}$ \\
Hypertension & $44(55.6 \%)$ & $33(68.8 \%)$ & 0.144 \\
Diabetes & $19(24.1 \%)$ & $11(22.9 \%)$ & 0.884 \\
Smoking & $42(53.2 \%)$ & $13(27.1 \%)$ & $0.004^{*}$ \\
Laboratory parameters: & & \\
TC $[\mathrm{mmol} / \mathrm{L}]$ & $4.91 \pm 1.1$ & $4.27 \pm 0.9$ & $0.001^{*}$ \\
TG $[\mathrm{mmol} / \mathrm{L}]$ & $1.40 \pm 1.3$ & $1.57 \pm 0.7$ & 0.435 \\
LDL $[\mathrm{mmol} / \mathrm{L}]$ & $3.09 \pm 0.9$ & $2.58 \pm 0.9$ & $0.004^{*}$ \\
HDL $[\mathrm{mmol} / \mathrm{L}]$ & $1.07 \pm 0.2$ & $1.12 \pm 0.3$ & 0.385 \\
WBC $\left[\times 10^{\circ} / \mathrm{L}\right]$ & $8.97 \pm 3.0$ & $6.56 \pm 1.8$ & $0.000^{*}$ \\
Thyroxine $[\mathrm{nmol} / \mathrm{L}]$ & $97.49 \pm 27.1$ & $104.16 \pm 23.4$ & 0.163 \\
RDW $[\%]$ & $13.47 \pm 1.0$ & $13.03 \pm 0.8$ & $0.008^{*}$ \\
CRP $[\mathrm{mg} / \mathrm{L}]$ & $23.61 \pm 19.5$ & $5.15 \pm 7.3$ & $0.000^{*}$ \\
sUA $[\mu \mathrm{mol} / \mathrm{L}]$ & $366.5 \pm 103.6$ & $362.3 \pm 91.4$ & 0.820 \\
\hline
\end{tabular}

Data presented as mean \pm standard deviation for variables; TC — total cholesterol; TG — triglycerides; LDL — low-density lipoprotein; HDL high-density lipoprotein; WBC - white blood count; RDW - red-cell distribution width; CRP - C-reactive protein; sUA — serum uric acid; ${ }^{*} \mathrm{p}<0.05$ AMI vs. control

lation of NLRP3 inflammasome activation. Interestingly, rheumatoid arthritis is coupled with increased incidence of myocardial infarction, and the severity of arthritis has been proven to be reduced in P2X7 receptor knockout mice [7].

This accumulating evidence suggests the possibility of an association between P2X7R and the development of atherosclerosis; however, the significance of its expression in clinical patients with AMI remains unclear. The present study was conducted to investigate the potential role of P2X7R in AMI.

\section{Methods}

\section{Study subjects}

Seventy-nine patients with AMI (ICD-9 codes 410), undergoing coronary angiography in the cardiac care unit of the First Affiliated Hospital of Wenzhou Medical College (China), were consecutively recruited for the present study (Table 1). AMI, including ST-segment elevation myocardial infarction (STEMI) and non-ST-segment elevation myocardial infarction (NSTEMI), was diagnosed based on the presence of two of the following criteria: prolonged chest pain; typical electrocardiogram changes; and increased troponin I level ( $>0.15$ $\mu \mathrm{g} / \mathrm{L})$. The control group comprised 48 patients recruited from the cardiology department. These patients presented chest discomfort and certain risk factors for coronary disease, but their coronary angiography results were normal. Exclusion criteria included patients with ischemic stroke history, peripheral vascular disease, hematological diseases, acute or chronic inflammation, liver dysfunction (2 times higher than the upper limit of the reference range of AST/ALT), severe renal dysfunction (chronic kidney disease stage: G4-5), autoimmune disease, and cancer.

Patients in both AMI and control groups were treated with dual antiplatelet therapy and statin as per standard protocol after hospitalization. Gensini scores were used to evaluate the severity of coronary stenotic lesions and was calculated by two technicians based on imaging results taken at different angles of the angiocardiography.

Written informed consent was obtained from all subjects before enrollment into this study. The study was approved by the Medical Ethics Committee of the First Affiliated Hospital of Wenzhou Medical College (No. 2017-145) and was performed in accordance with the Declaration of Helsinki.

All patients underwent clinical follow-up for one complete year after discharge, to determine the end point based on the findings at the last visit or last telephone call. However, some patients were lost during follow-up for various reasons. The composite end point was major adverse cardiovascular events (MACE), which included allcause death, myocardial infarction, target lesion 
revascularization, heart failure, and recurrent angina, which were identified through phone-calls or hospitalization.

\section{Sample collection}

In the AMI group, emergency coronary angiography or percutaneous coronary intervention was performed within $12 \mathrm{~h}$ of symptom onset, and blood samples were collected immediately after the procedure. In the control group, blood samples were obtained immediately after coronary angiography following their admission. Peripheral venous blood was drawn from the antecubital vein while the patients were in a fasting state. Blood was collected with EDTA as an anticoagulant. Peripheral blood mononuclear cells (PBMCs) were immediately isolated from peripheral venous blood using Ficoll density gradient centrifugation.

\section{Quantitative real-time PCR}

RNA extraction from PBMCs was performed according to manufacturer protocol (using TRIzol, Invitrogen). Synthesis of cDNAs was performed by reverse transcription with $2 \mu \mathrm{g}$ of total RNA using a cDNA Transcription kit (Thermo Fisher Scientific), as described by the manufacturer. Real-time polymerase chain reactions (PCR) were performed by SYBR Premix Ex Taq Kit (TaKaRa, Japan) according to manufacturer instructions. The PCR reactions were evaluated by the ABI-7500 Sequence Detection System. The primer sequences used in the study were as follows: P2X7R (NM 002562.5), forward: 5'-GAACAATATCGACTTC $\bar{C} C \mathrm{CGG}-3$ ', reverse: 5'-TTATCGCCTGTTTCTCGGAAG-3'; GAPDH (NM_001256799.2), forward: 5'-CAGGAGGCATTGCTGATGAT-3', reverse: 5'-GAAGGCTGGGGCTCATTT-3'. Values were normalized to that of the housekeeping gene GAPDH. According to manufacturer guidelines, the ${ }^{\Delta \Delta} \mathrm{Ct}$ method was used to determine relative expression levels. Statistical analyses were performed using ${ }^{\Delta \Delta} \mathrm{Ct}$ values.

\section{Statistical analysis}

Statistical analysis was performed using SPSS v.20 software (SPSS Inc., Chicago, IL). All clinical data were represented as mean \pm standard deviation for continuous variables, and as number (\%) for incidence rates. Independent-samples $\mathrm{T}$ test or the Wilcoxon Rank Sum Test was used for comparison of the two groups of individuals. Chi-square test was used to compare proportions. The association between relative levels of P2X7R and follow-up endpoints was ascertained by the Kaplan-Meier analysis. Receiver operating characteristic (ROC)

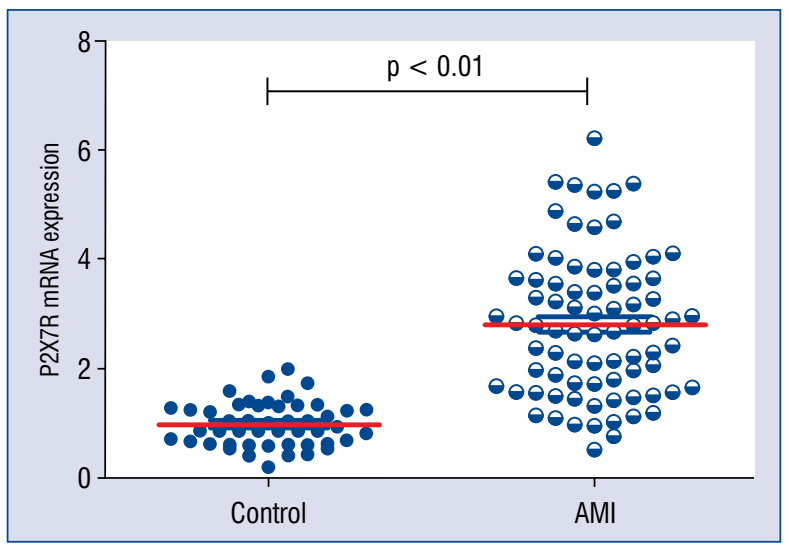

Figure 1. Circulating P2X7R mRNA expression levels in the acute myocardial infarction (AMI) and control groups. Data are presented as the mean \pm standard error of the mean; $p<0.01$ compared to the control.

curve analysis was performed to assess P2X7R as a predictor for distinguishing AMI from non-AMI. Correlations between variables were determined with the Spearman test. The univariate and multivariate cox regression analysis was performed to assess P2X7R expression levels and risk of MACE after myocardial infarction. In all tests, a value of $\mathrm{p}<0.05$ was considered statistically significant.

\section{Results}

\section{Baseline characteristics of patients}

The baseline characteristics of patients are shown in Table 1 . There were 73 cases of STEMI and 6 cases of NSTEMI in the AMI group. Among them, 76 cases were treated with emergency PCI and 3 cases were treated with elective CABG after coronary angiography. No statistical difference in age, hypertension, diabetes, triglycerides, highdensity lipoprotein, thyroxine, and uric acid was observed between the two groups. The AMI group had higher values of male-to-female ratio, proportion of smoking, total cholesterol, low-density lipoprotein (LDL), white blood count, red cell distribution width, and $\mathrm{C}$-reactive protein (CRP) $(\mathrm{p}<0.01)$ than the control group.

\section{P2X7R mRNA expression levels}

The P2X7R mRNA expression in PBMCs of 127 blood samples, determined by quantitative real-time PCR, is shown in Figure 1. The AMI group had 2.85-fold higher P2X7R mRNA expression than the control group ( $2.81 \pm 0.15$ vs. $0.99 \pm$ $\pm 0.06, \mathrm{p}<0.01$, Fig. 1). 


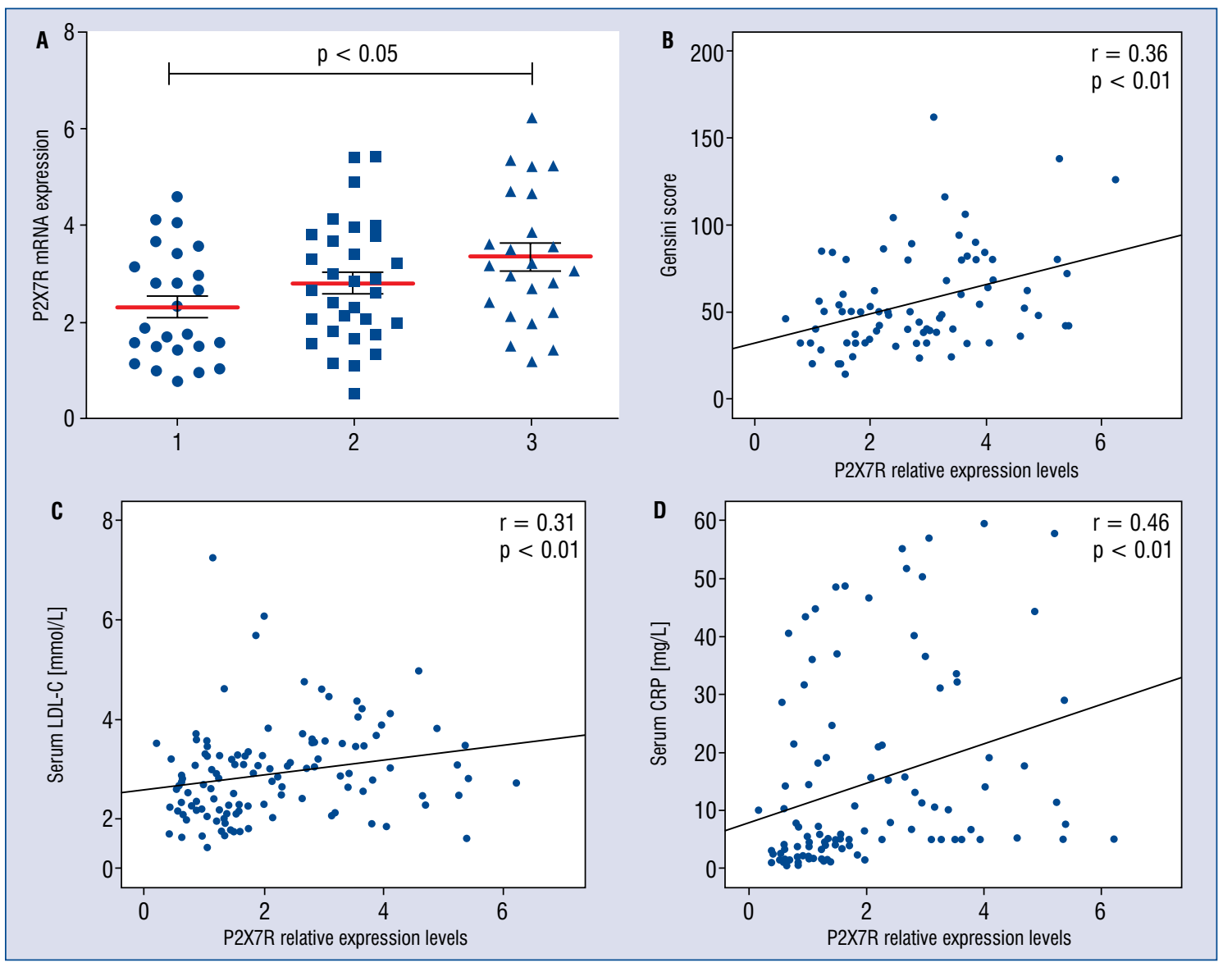

Figure 2. Pattern of circulating P2X7R expression levels in patients with acute myocardial infarction (AMI); A. Relative P2X7R levels in patients with 1, 2, or 3 affected branches ( $n=1,2$, and 3); red lines represent the mean values; B. Correlations between plasma P2X7R levels and coronary Gensini scores in patients with AMI $(r=0.36, p<0.01)$;

C. Correlations between plasma P2X7R levels and serum low-density lipoprotein cholesterol (LDL-C);

D. Correlations between plasma P2X7R levels and serum C-reactive protein (CRP).

\section{P2X7R mRNA levels and stenosis}

As shown in Figure 2A, significantly higher P2X7R mRNA expression was detected in patients with AMI with three-vessel lesions than in patients with single-vessel lesions $(p<0.05)$. A significant Spearman correlation coefficient was noted between angiographic Gensini scores and circulating P2X7R mRNA expression levels $(r=0.36, p<0.01$, Fig. 2B). Additionally, P2X7R mRNA expression levels were found to be positively correlated with serum LDL and CRP values $(r=0.31$ and 0.46, $p<0.05$; Fig. $2 \mathrm{C}, \mathrm{D})$.

\section{The diagnostic ability of P2X7R in AMI}

$\mathrm{ROC}$ analysis was performed to determine the diagnostic ability of P2X7R in AMI. The ROC curves of P2X7R mRNA expression levels in PBMCs could distinguish between the AMI and control groups with an area under the curve (AUC) of 0.928 (95\% confidence interval [CI] 0.885-0.971, $\mathrm{p}<0.01$, Fig. 3). The ROC curves revealed that the cut-off value of P2X7R mRNA expression in PBMCs differentiating patients with AMI from control subjects was 1.418 .

\section{P2X7R mRNA and MACE}

Patients enrolled in this study were followed up for 360 days. Fourteen patients were lost to follow-up. All subjects were divided into two groups according to fold changes of P2X7R mRNA by dichotomization: the lower level (0.52-2.68-fold changes) and the higher level (2.69-5.42-fold changes). The end point incidence significantly differed according to P2X7R expression levels, as analyzed using the Kaplan-Meier curve $\left(\chi^{2}=5.29\right.$, $\mathrm{p}=0.021$; Fig. 4). The univariate and multivariate Cox regression analysis revealed that P2X7R expression levels significantly increased the risk 


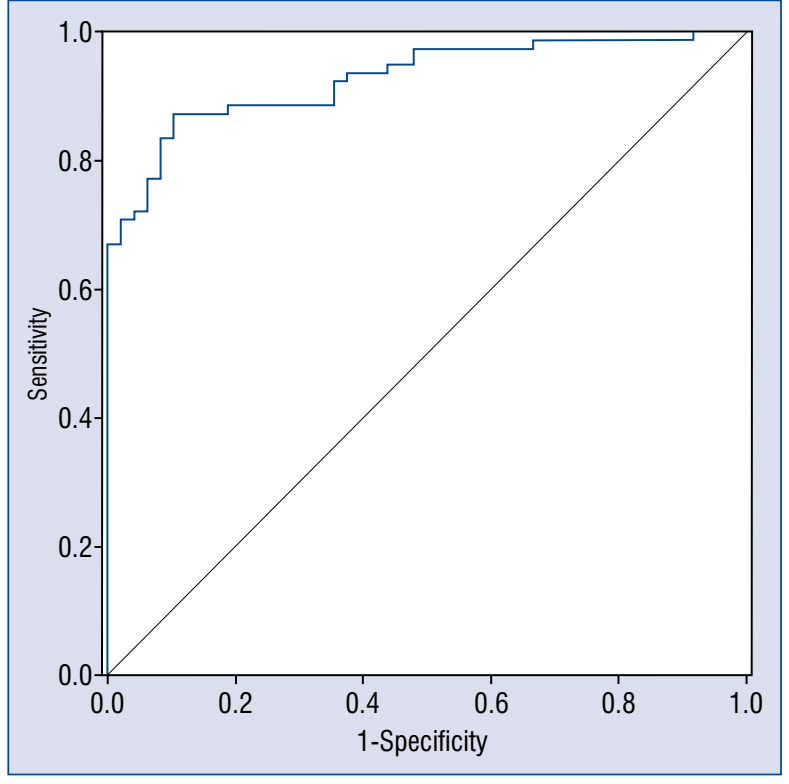

Figure 3. Receiver operating characteristic curve analysis of P2X7R expression level in peripheral blood mononuclear cells to predict acute myocardial infarction.

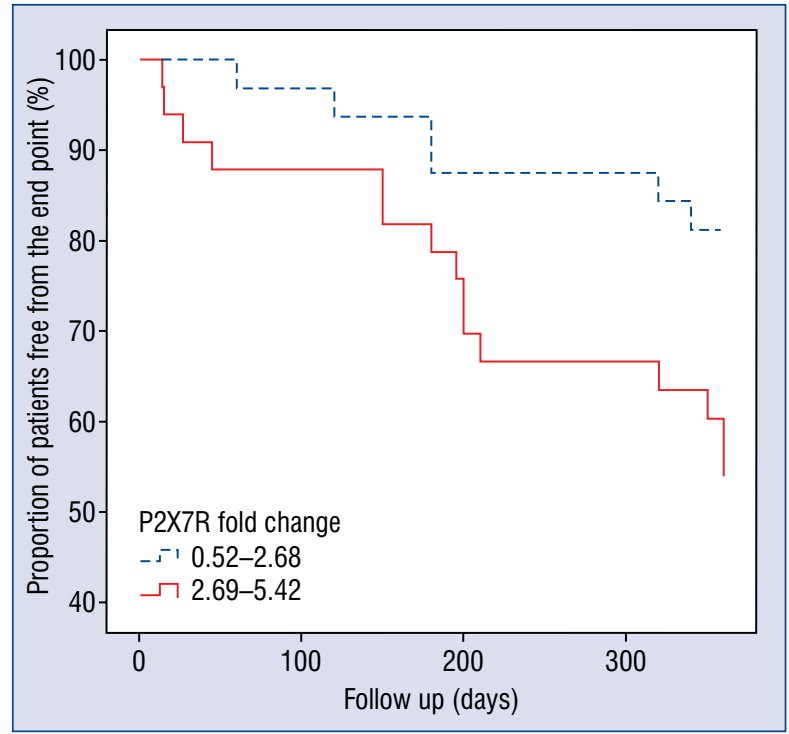

Figure 4. Kaplan-Meier curve analysis of patients free of end point after acute myocardial infarction, according to P2X7R mRNA expression level. The end point was defined as all-cause death, myocardial infarction, target lesion revascularization, heart failure, and recurrent angina.

Table 2. The correlations of the P2X7R mRNA expression level with major adverse cardiovascular events after acute myocardial infarction using the uni and multivariate Cox analysis.

\begin{tabular}{lccccc}
\hline \multirow{2}{*}{ Factors } & \multicolumn{2}{c}{ Univariate Cox } & & & \multicolumn{2}{c}{ Multivariate Cox } \\
\cline { 2 - 3 } \cline { 5 - 6 } & HR $(95 \% \mathrm{Cl})$ & $\mathbf{P}$ & & HR (95\% Cl) & P \\
\hline Age & $1.01(0.98-1.04)$ & 0.640 & & $1.00(0.96-1.05)$ & 0.860 \\
Sex & $0.43(0.25-1.83)$ & 0.669 & & $0.46(0.11-1.96)$ & 0.294 \\
Hypertension & $5.09(1.50-17.32)$ & 0.009 & & $3.60(1.02-12.74)$ & 0.047 \\
Diabetes & $2.39(1.01-5.69)$ & 0.049 & & $1.26(0.46-3.41)$ & 0.652 \\
Smoking & $1.30(0.55-3.10)$ & 0.547 & & $1.68(0.53-5.29)$ & 0.380 \\
Total cholesterol & $0.91(0.61-1.35)$ & 0.632 & & $1.04(0.36-3.01)$ & 0.942 \\
LDL-C & $0.83(0.50-1.37)$ & 0.465 & & $0.74(0.20-2.75)$ & 0.652 \\
Higher P2X7R level & $2.87(1.11-7.42)$ & $0.029 *$ & & $3.28(1.12-9.65)$ & $0.031^{*}$ \\
\hline
\end{tabular}

*Patients with a lower level of P2X7R mRNA expression (0.52-2.68-fold changes) were set as the reference group. $\mathrm{Cl}-\mathrm{confidence}$ interval; HR - hazard ratio; LDL-C — low-density lipoprotein; higher P2X7R level, 2.69-5.42-fold changes of P2X7R mRNA expression level

of MACE after myocardial infarction (hazard ratio [HR] 2.87, 95\% CI 1.11-7.42 and HR 3.28, 95\% CI 1.12-9.65; Table 2).

\section{Discussion}

Atherosclerosis is thought to be the primary cause of AMI. Initiation, progression, and complications of atherosclerotic plaque are considered to be a result of the complex phenomena involving the interplay of lipoproteins, vascular wall components, blood cells, and the immune system. Multiple levels of evidence support a close relationship between inflammation and atherosclerosis.

$\mathrm{P} 2 \mathrm{X} 7 \mathrm{R}$ is considered to play dual roles in several inflammatory pathological conditions [8]. P2X7R functions as a second signal during NLRP3 inflammasome activation and interleukin (IL)- $1 \beta$ release. It contributes to activation of effector $\mathrm{T}$ cells, favors polarization of $\mathrm{T}$ cells into Th17 cells, and reduces suppressive activity and viability of Tregs. On the other hand, P2X7R can induce an 
excessive production and release of inflammatory mediators coupled to a high incidence of apoptotic and necrotic cell death due the release of large amounts of ATP, which causes a self-sustained pro-inflammatory deleterious cycle.

In the present study, it was found that P2X7R mRNA expression levels were elevated in PBMCs of patients with AMI, and evaluated the diagnostic ability of P2X7R using ROC analyses. The AUC for P2X7R was 0.928 (95\% CI 0.885-0.971) for differentiating between patients with AMI from control subjects. Furthermore, the indicated P2X7R was positively correlated with severity of coronary artery stenosis, and was linearly correlated with serum LDL and CRP. Based on a current understanding, P2X7R clearly plays a key role in cardiovascular physiology and pathophysiology. It shows deleterious effects in cardiovascular diseases by promoting inflammation, thrombosis, and endothelial dysfunction [9]. Its activation promotes the assembly of NLRP3 inflammasome and an unconventional release of pro-inflammatory leaderless cytokines IL- $1 \beta$ and IL-18 [10]. A previous study illustrated that inhibition of P2X7R may be able to suppress the AMPK/MAPK signaling pathway and consequently downregulate both EMMPRIN and MMP-9 expression in monocyte-derived macrophages, which correlated with advanced atherosclerotic lesions, followed by plaque rupture and myocardial infarction [11]. Furthermore, Stachon et al. [12] found that P2X7 receptor is highly expressed in murine atherosclerotic lesions, particularly in lesioned macrophages, and P2X7-deficient mice showed smaller atherosclerotic lesions than P2X7-competent mice after 16 weeks of a high cholesterol diet. All of these effects together suggest that P2X7R, indeed participates in the development of atherosclerosis.

However, data on the relationship between P2X7R and MACE after AMI remains insufficient. The inhibition of P2X7 receptor attenuated sympathetic nerve sprouting after myocardial infarction via the NLRP3/IL-1 $\beta$ pathway, which contributes to neural and cardiac remodeling [13]. Microglial P2X7R in rat hypothalamic paraventricular nuclei regulate the sympathoexcitatory responses in AMI [14]. NONRATT021972 siRNA decreases the upregulation of P2X7R and improves cardiac function after myocardial ischemia [15]. According to available research, this is the first report to propose that a higher expression of P2X7R mRNA is independently associated with higher rate of MACE within 360 days of AMI. Although inflammatory markers, such as hs-CRP, have been shown to identify patients with increased risk for incident coronary heart disease [16], but there were no significant associations between hs-CRP levels after AMI and their symptoms, function and quality of life after adjusting for prior health status [17]. Giuliani et al. [18] found correlation of P2X7R and CRP was widely different depending on the disease and best correlation was found in patients suffering of ischemia. So P2X7R measurement might complement that of CRP in a differential diagnosis of inflammatory conditions of different etiology.

\section{Limitations of the study}

Nevertheless, this study did have several limitations. Firstly, it was a single-center study with a relatively small study population; changes in longterm clinical outcomes may need to be confirmed by increasing both the total number of patients and the duration of follow-up. Secondly, this study lacked cardiac remodeling data during the follow-up period; therefore, further studies are warranted. Thirdly, measurement of P2X7 protein was lacking which should be required in further study.

\section{Conclusions}

The present study identified that the purinergic receptor P2X7R was elevated in AMI patients and was closely associated with the severity of coronary artery stenosis and prognosis of AMI.

\section{Funding}

This study was supported by the Bureau of traditional Chinese Medicine of Zhejiang Province (No. 2016ZA137 and No. LQ15H020005), the National Natural Science Foundation of China (No. 81670224).

\section{Conflict of interest: None declared}

\section{References}

1. Packard R, Lichtman A, Libby P. Innate and adaptive immunity in atherosclerosis. Sem Immunopathol. 2009; 31(1): 5-22, doi: 10.1007/s00281-009-0153-8.

2. Hansson GK. Inflammation, atherosclerosis, and coronary artery disease. N Engl J Med. 2005; 352(16): 1685-1695, doi: 10.1056/ NEJMra043430, indexed in Pubmed: 15843671.

3. Newby AC, George SJ, Ismail Y, et al. Vulnerable atherosclerotic plaque metalloproteinases and foam cell phenotypes. Thromb Haemost. 2009; 101(6): 1006-1011, indexed in Pubmed: 19492140.

4. Baroja-Mazo A, Pelegrín P. Modulating P2X7 receptor signaling during rheumatoid arthritis: new therapeutic approaches for bisphosphonates. J Osteoporos. 2012; 2012: 408242, doi: 10.1155/2012/408242, indexed in Pubmed: 22830074. 
5. Piscopiello M, Sessa M, Anzalone N, et al. P2X7 receptor is expressed in human vessels and might play a role in atherosclerosis. Int J Cardiol. 2013; 168(3): 2863-2866, doi: 10.1016/j. ijcard.2013.03.084, indexed in Pubmed: 23602287.

6. Peng K, Liu L, Wei D, et al. P2X7R is involved in the progression of atherosclerosis by promoting NLRP3 inflammasome activation. Int J Mol Med. 2015; 35(5): 1179-1188, doi: 10.3892/ ijmm.2015.2129, indexed in Pubmed: 25761252.

7. Labasi JM, Petrushova N, Donovan C, et al. Absence of the P2X7 receptor alters leukocyte function and attenuates an inflammatory response. J Immunol. 2002; 168(12): 6436-6445, doi: 10.4049/ jimmunol.168.12.6436, indexed in Pubmed: 12055263.

8. Savio L, Mello P, Silva C, et al. The P2X7 receptor in inflammatory diseases: angel or demon? Front Pharmacol. 2018; 9, doi: 10.3389/fphar.2018.00052.

9. Burnstock G. Purinergic signaling in the cardiovascular system. Circulation Research. 2017; 120(1): 207-228, doi: 10.1161/circresaha.116.309726.

10. Mariathasan S, Weiss DS, Newton K, et al. Cryopyrin activates the inflammasome in response to toxins and ATP. Nature. 2006; 440(7081): 228-232, doi: 10.1038/nature04515, indexed in Pubmed: 16407890 .

11. Lin Lu, Huang S, Zhu Z, et al. P2X7 receptor regulates EMMPRIN and MMP9 expression through AMPK/MAPK signaling in PMA induced macrophages. Mol Med Rep. 2018; 18(3): 3027-3033, doi: 10.3892/mmr.2018.9282, indexed in Pubmed: 30015874 .

12. Stachon P, Heidenreich A, Merz J, et al. P2X deficiency blocks lesional inflammasome activity and ameliorates atherosclerosis in mice. Circulation. 2017; 135(25): 2524-2533, doi: 10.1161/CIRCULATIONAHA.117.027400, indexed in Pubmed: 28377486.

13. Yin J, Wang Yu, Hu H, et al. P2X receptor inhibition attenuated sympathetic nerve sprouting after myocardial infarction via the NLRP3/LL-1 $\beta$ pathway. J Cell Mol Med. 2017; 21(11): 2695-2710, doi: 10.1111/jcmm.13185, indexed in Pubmed: 28470940.

14. Du D, Jiang M, Liu M, et al. Microglial P2X receptor in the hypothalamic paraventricular nuclei contributes to sympathoexcitatory responses in acute myocardial infarction rat. Neurosci Lett. 2015; 587: 22-28, doi: 10.1016/j.neulet.2014.12.026, indexed in Pubmed: 25524407.

15. Tu G, Zou L, Liu S, et al. Long noncoding NONRATT021972 siRNA normalized abnormal sympathetic activity mediated by the upregulation of $\mathrm{P} 2 \mathrm{X} 7$ receptor in superior cervical ganglia after myocardial ischemia. Purinergic Signal. 2016; 12(3): 521-535, doi: 10.1007/s11302-016-9518-3, indexed in Pubmed: 27215605.

16. Kaptoge S, Di Angelantonio E, Lowe G, et al. C-reactive protein concentration and risk of coronary heart disease, stroke, and mortality: an individual participant meta-analysis. Lancet. 2010; 375(9709): 132-140, doi: 10.1016/S0140-6736(09)61717-7, indexed in Pubmed: 20031199.

17. Pokharel Y, Sharma PP, Qintar M, et al. High-sensitivity C-reactive protein levels and health status outcomes after myocardial infarction. Atherosclerosis. 2017; 266: 16-23, doi: 10.1016/j. atherosclerosis.2017.09.019, indexed in Pubmed: 28946036.

18. Giuliani AL, Berchan M, Sanz JM, et al. The P2X7 receptor is shed into circulation: correlation with C-reactive protein levels. Front Immunol. 2019; 10: 793, doi: 10.3389/fimmu.2019.00793, indexed in Pubmed: 31031771. 